Pacific Journal of Mathematics

CANCELLATION OF LOW-RANK VECTOR BUNDLES 


\title{
CANCELLATION OF LOW-RANK VECTOR BUNDLES
}

\author{
K. R. GOODEARL
}

\begin{abstract}
The purpose of this paper is to show that even though vector bundles cannot in general be cancelled from direct sums (Whitney sums), in certain low-rank situations vector bundles can be cancelled at the expense of complexifying or quaternionifying the remaining terms. To be specific, let $\lambda, \xi_{1}, \xi_{2}$ be vector bundles over a paracompact space $X$, such that $\lambda \oplus \xi_{1} \cong \lambda \oplus \xi_{2}$. First assume that these are real vector bundles. If $\lambda$ is a line bundle of finite type, then the complexifications of $\xi_{1}$ and $\xi_{2}$ are isomorphic, and hence $2 \xi_{1} \cong 2 \xi_{2}$ (where $2 \xi_{1}$ denotes the direct sum of two copies of $\xi_{i}$ ), while if $\lambda$ is a direct sum of two line bundles of finite type, then the quaternionifications of $\xi_{1}$ and $\xi_{2}$ are isomorphic, and hence $4 \xi_{1} \cong 4 \xi_{2}$. Now assume that these are complex vector bundles. If $\lambda$ is the complexification of a real line bundle of finite type (in particular, $\lambda$ could be a trivial complex vector bundle of rank 1), then the quaternionifications of $\xi_{1}$ and $\xi_{2}$ are isomorphic, and hence $\xi_{1} \oplus \bar{\xi}_{1} \cong \xi_{2} \oplus \bar{\xi}_{2}$

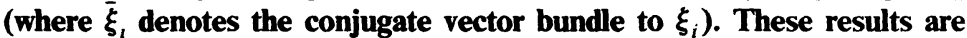
independent of the dimension of the space $X$, and also independent of the dimensions of the fibres of $\xi_{1}$ and $\xi_{2}$. The same results also hold for smooth vector bundles over a smooth manifold.
\end{abstract}

I. Background on vector bundles. Let $\mathbf{F}$ be one of $\mathbf{R}, \mathbf{C}$, or $\mathbf{H}$. We work with $\mathbf{F}$-vector bundles over a topological space $X$ in the same generality as Swan [9], so that we do not require the dimensions of the fibres of a vector bundle $\xi$ to be constant. (However, the local triviality condition on $\xi$ forces the fibre-dimension of $\xi$ to be locally constant, and hence the fibre-dimension will be constant if $X$ is connected.) A vector bundle $\xi$ over $X$ is said to be of finite type provided that there exists a finite open covering $U_{1}, \ldots, U_{n}$ of $X$ such that the restriction of $\xi$ to each $U_{i}$ is trivial. (In particular, this places a bound on the fibre-dimension of $\xi$.) Of course if $X$ is compact, then all vector bundles over $X$ are of finite type.

The basic mechanism for algebraic investigations of $\mathbf{F}$-vector bundles over $X$ is the relationship between such vector bundles and modules over the ring $C(X, \mathbf{F})$ of continuous $\mathbf{F}$-valued functions on $X$. This relationship is effected through the section functor $\Gamma$, which assigns to each $F$-vector bundle $\xi$ the $C(X, \mathbf{F})$-module $\Gamma(\xi)$ of continuous sections of $\xi$, and which assigns to each $\mathbf{F}$-vector bundle map $f: \xi \rightarrow \eta$ the induced $C(X, \mathbf{F})$-module homomorphism $\Gamma(f): \Gamma(\xi) \rightarrow \Gamma(\eta)$. (In the case where $\mathbf{F}=\mathbf{H}$, a choice of right versus left vector spaces and modules is required for consistency. As we prefer to write homomorphisms on the left, let us stipulate that all our 
$\mathbf{H}$-vector spaces are right vector spaces, and that all our $C(X, \mathbf{H})$-modules are right modules.) An obvious property of the section functor $\Gamma$ is that it preserves direct sums, that is, $\Gamma(\xi \oplus \eta) \cong \Gamma(\xi) \oplus \Gamma(\eta)$. The additional properties of $\Gamma$ which we shall need may be outlined as follows.

TheOREM 1.1. Let $\mathbf{F}$ be one of $\mathbf{R}, \mathbf{C}$, or $\mathbf{H}$, let $X$ be a paracompact topological space, and let $\xi, \eta$ be $\mathbf{F}$-vector bundles over $X$.

(a) Given any $C(X, \mathbf{F})$-module homomorphism $f: \Gamma(\xi) \rightarrow \Gamma(\eta)$, there exists a unique F-vector bundle map $g: \xi \rightarrow \eta$ such that $\Gamma(g)=f$.

(b) $\xi \cong \eta$ if and only if $\Gamma(\xi) \cong \Gamma(\eta)$.

(c) If $\xi$ is of finite type, then $\Gamma(\xi)$ is a finitely generated projective $C(X, \mathbf{F})$-module.

(d) If $A$ is a finitely generated projective $C(X, \mathbf{F})$-module, then there exists an $\mathbf{F}$-vector bundle $\zeta$ over $X$ of finite type such that $\Gamma(\zeta) \cong A$.

Proof. (a), (b) These properties hold more generally for vector bundles over a normal topological space [9, Theorem 1, Corollary 4].

(c) We need to know that there exists an F-vector bundle $\zeta$ over $X$ such that $\xi \oplus \zeta$ is trivial. In case the fibre-dimension of $\xi$ is constant, this is proved in [7, Chapter 3, Proposition 5.8]. In general, since the fibre-dimension of $\xi$ is locally constant, and also bounded (because $\xi$ is of finite type), $X$ may be expressed as a disjoint union of clopen sets $U_{1}, \ldots, U_{n}$ such that the restriction of $\xi$ to each $U_{i}$ has constant fibre-dimension. Applying the theorem above to each of these restrictions then provides us with the desired vector bundle $\zeta$. Now $\Gamma(\xi) \oplus \Gamma(\zeta)$ is a free $C(X, \mathbf{F})$-module of finite rank, and hence $\Gamma(\xi)$ is a finitely generated projective $C(X, \mathbf{F})$-module.

(d) The proof of [9, Theorem 2] may be used to show that there exists an $\mathbf{F}$-vector bundle $\zeta$ over $X$ such that $\Gamma(\zeta) \cong A$ and $\zeta$ is a direct summand of a trivial bundle. In particular, it follows that the fibre-dimension of $\zeta$ is bounded. Applying [7, Chapter 3, Proposition 5.8] to clopen subsets of $X$ as above, we conclude that $\zeta$ must be of finite type.

In particular, it follows from Theorem 1.1 that over a paracompact space $X$, the section functor $\Gamma$ provides a category equivalence between the category of $\mathbf{F}$-vector bundles over $X$ of finite type and the category of finitely generated projective $C(X, \mathbf{F})$-modules.

We denote the complexification of a real vector bundle $\xi$ by $\xi^{\mathbf{C}}$, and we denote the quaternionification of $\xi$ by $\xi^{\mathbf{H}}$. Note that as real vector bundles, $\xi^{\mathbf{C}} \cong 2 \xi$ (the direct sum of two copies of $\xi$ ), and $\xi^{\mathbf{H}} \cong 4 \xi$. We 
denote the quaternionification of a complex vector bundle $\eta$ by $\eta^{\mathbf{H}}$. Note

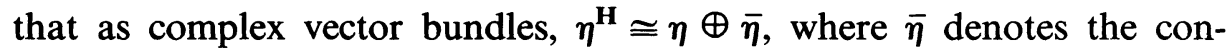
jugate vector bundle of $\eta$. Complexification and quaternionification commute with the section functor in the following straightforward manner, the details of which we leave to the reader. If, for instance, $\xi$ is a real vector bundle over a space $X$, then the $C(X, \mathbf{R})$-module $\Gamma(\xi)$ may be extended to a $C(X, \mathbf{C})$-module by passing to the tensor product $\Gamma(\xi) \otimes_{C(X, \mathbf{R})} C(X, \mathbf{C})$. This tensor product is naturally isomorphic to $\Gamma(\xi) \otimes_{\mathbf{R}} \mathbf{C}$, because $\{1, i\}$ is simultaneously a basis for $\mathbf{C}$ as an $\mathbf{R}$-vector space and for $C(X, \mathbf{C})$ as a free $C(X, \mathbf{R})$-module. Similarly, we may identify $\Gamma(\xi) \otimes_{C(X, \mathbf{R})} C(X, \mathbf{H})$ with $\Gamma(\xi) \otimes_{\mathbf{R}} \mathbf{H}$, and given a complex vector bundle $\eta$ over $X$, we may identify $\Gamma(\eta) \otimes_{C(X, C)} C(X, \mathbf{H})$ with $\Gamma(\eta) \otimes_{C} \mathbf{H}$.

Proposition 1.2. Let $X$ be a topological space.

(a) If $\xi$ is a real vector bundle over $X$, then $\Gamma\left(\xi^{\mathbf{C}}\right) \cong \Gamma(\xi) \otimes_{\mathbf{R}} \mathbf{C}$ as $C(X, \mathbf{C})$-modules, and $\Gamma\left(\xi^{\mathbf{H}}\right) \cong \Gamma(\xi) \otimes_{\mathbf{R}} \mathbf{H}$ as $C(X, \mathbf{H})$-modules.

(b) If $\eta$ is a complex vector bundle over $X$, then $\Gamma\left(\eta^{\mathbf{H}}\right) \cong \Gamma(\eta) \otimes_{\mathbf{C}} \mathbf{H}$ as $C(X, \mathbf{H})$-modules.

Proposition 1.3. Let $\mathbf{F}$ be either $\mathbf{R}$ or $\mathbf{C}$, and let $X$ be a paracompact topological space. Let $\lambda$ be an F-vector bundle over $X$, and let $\varphi$ be the natural ring homomorphism of $C(X, \mathbf{F})$ into the endomorphism ring of the $C(X, \mathbf{F})$-module $\Gamma(\lambda)$. If $\lambda$ is a line bundle of finite type, then $\varphi$ is an isomorphism.

Proof. Set $R=C(X, \mathbf{F})$ and $E=\operatorname{End}_{R}(\Gamma(\lambda))$. Since $\lambda$ is of finite type, Theorem 1.1 shows that $\Gamma(\lambda)$ is a finitely generated projective $R$-module.

For $x \in X$, let $M(x)$ denote the maximal ideal of $R$ consisting of all functions in $R$ that vanish at $x$. By [9, Corollary 3], $\Gamma(\lambda) / M(x) \Gamma(\lambda)$ is isomorphic to the fibre of $\lambda$ at $x$, which is a line. Consequently, the localized module $\Gamma(\lambda)_{M(x)}$ is a rank 1 free module. As the intersection of the maximal ideals of the form $M(x)$ is zero, the set of these maximal ideals is dense (in the Zariski topology) in $\operatorname{Spec}(R)$. In addition, the map which assigns to each $P \in \operatorname{Spec}(R)$ the rank of the free $R_{P}$-module $\Gamma(\lambda)_{P}$ is locally constant [2, Chapter II, §5.2, Theorem 1]. As a result, $\Gamma(\lambda)_{M}$ is a rank 1 free $R_{M}$-module for every maximal ideal $M$ of $R$.

It now follows that for each maximal ideal $M$ of $R$, the localization of $\varphi$ at $M$ is an isomorphism of $R_{M}$ onto $E_{M}$ (which can be identified with the endomorphism ring of $\Gamma(\lambda)_{M}$ because $\Gamma(\lambda)$ is a finitely generated projective module). Therefore $\varphi$ is an isomorphism. 
The analogs of Theorem 1.1 and Propositions 1.2 and 1.3 hold for smooth F-vector bundles over a smooth manifold $X$, in which case $C(X, \mathbf{F})$ is replaced by the ring $C^{\infty}(X, \mathbf{F})$ of smooth $\mathbf{F}$-valued functions on $X$. For proofs of the relevant properties in the case of smooth vector bundles of constant fibre-dimension, see [6, $\S 2.14,2.23,2.24]$.

II. Background on cancellation. The standard cancellation results for vector bundles over a topological space $X$ require that one of the vector bundles involved have fibre-dimension everywhere greater than the dimension of $X$. For example, let $X$ be an $n$-dimensional CW-complex, and let $\theta, \xi_{1}, \xi_{2}$ be real vector bundles over $X$ such that $\theta$ is trivial and $\theta \oplus \xi_{1} \cong \theta \oplus \xi_{2}$. If the fibres of $\xi_{1}$ have dimension at least $n+1$, then $\xi_{1} \cong \xi_{2}$ [7, Chapter 8, Theorem 1.5]. Even if the fibres of $\xi_{1}$ have small dimension, those of $(n+1) \xi_{1}$ have large enough dimension, and hence $(n+1) \xi_{1} \cong(n+1) \xi_{2}$.

We are interested in cancellation results of the form

$$
\eta \oplus \xi_{1} \cong \eta \oplus \xi_{2} \Rightarrow r \xi_{1} \cong r \xi_{2}
$$

in which the positive integer $r$ does not depend on the dimension of the base space. One such result is immediate from the following theorem of T. Y. Lam. To keep our module notation in line with our vector bundle notation, we write $s M$ to denote the direct sum of $s$ copies of a module $M$.

TheOREM 2.1. [ Lam] Let $R$ be a commutative ring, and let $A, B_{1}, B_{2}$ be $R$-modules such that $A \oplus B_{1} \cong A \oplus B_{2}$. Assume that $A$ is free of rank $n$ and that $B_{2}$ is free of rank $k$, for some positive integers $n, k$. Then $r B_{1} \cong r B_{2}$ for all integers $r \geq n+(n / k)$.

\section{Proof. [8, Theorem 2].}

COROLlaRY 2.2. Let $X$ be a paracompact topological space, and let $\theta$, $\xi_{1}, \xi_{2}$ be real or complex vector bundles over $X$ such that $\theta \oplus \xi_{1} \cong \theta \oplus \xi_{2}$. Assume that $\theta$ is trivial of rank $n$ and that $\xi_{2}$ is trivial of rank $k$, for some positive integers $n, k$. Then $r \xi_{1} \cong r \xi_{2}$ for all integers $r \geq n+(n / k)$.

Proof. Theorems 1.1 and 2.1.

In particular, Corollary 2.2 implies that if $\theta, \xi_{1}, \xi_{2}$ are real or complex vector bundles over a paracompact space satisfying $\theta \oplus \xi_{1} \cong \theta \oplus \xi_{2}$, and if $\theta, \xi_{2}$ are trivial with $\theta$ having rank $n$, then $r \xi_{1} \cong r \xi_{2}$ for all integers $r \geq 2 n$. While this conclusion is open if $\theta$ and $\xi_{2}$ are allowed to be 
non-trivial, we can at least guarantee that $r \xi_{1} \cong r \xi_{2}$ for some positive integers $r$, as follows.

TheOREM 2.3. Let $A, B_{1}, B_{2}$ be finitely generated projective modules over a commutative ring $R$. If $A \oplus B_{1} \cong A \oplus B_{2}$, then there exists a positive integer $s$ such that $r B_{1} \cong r B_{2}$ for all integers $r \geq s$.

Proof. The modules $A, B_{1}, B_{2}$ and the inverse isomorphisms between $A \oplus B_{1}$ and $A \oplus B_{2}$ may be defined in terms of a finite set of matrices over $R$. Let $S$ be the subring of $R$ generated by the entries of these matrices. Then there exist finitely generated projective $S$-modules $C, D_{1}$, $D_{2}$ such that $C \otimes_{S} R \cong A$ and each $D_{i} \otimes_{S} R \cong B_{i}$, while also $C \oplus D_{1} \cong$ $C \oplus D_{2}$. Thus there is no loss of generality in assuming that $R$ is a finitely generated ring. Now $R$ is a homomorphic image of a polynomial ring over $\mathbf{Z}$ in finitely many indeterminates, and hence $R$ is a noetherian ring with finite Krull dimension $d$.

Because of [2, Chapter II, §5.2, Theorem 1], there is a ring decomposition $R=R^{\prime} \times R^{\prime \prime}$ such that $R^{\prime} B_{1}=0$, while $\left(R^{\prime \prime} B_{1}\right)_{P} \neq 0$ for all prime ideals $P$ of $R^{\prime \prime}$. Hence, we need only consider the cases in which either $B_{1}=0$ or else $B_{1}$ is nonzero at all localizations. If $B_{1}=0$, then $A \cong$ $A \oplus B_{2}$, and by localization we find that $B_{2}=0$. Therefore there is no loss of generality in assuming that $\left(B_{1}\right)_{P} \neq 0$ for all prime ideals $P$ of $R$.

Now given an integer $r \geq d+1$, for each maximal ideal $M$ of $R$ the free $R_{M}$-module $r\left(B_{1}\right)_{M}$ has rank at least $d+1$. Applying the Bass Cancellation Theorem [1, Chapter IV, Corollary 3.5] to the relation

$$
r A \oplus r B_{1} \cong r A \oplus r B_{2},
$$

we conclude that $r B_{1} \cong r B_{2}$.

COROLlary 2.4. Let $X$ be a paracompact topological space, and let $\eta$, $\xi_{1}, \xi_{2}$ be real or complex vector bundles over $X$, of finite type. If $\eta \oplus \xi_{1} \cong$ $\eta \oplus \xi_{2}$, then there exists a positive integer s such that $r \xi_{1} \cong r \xi_{2}$ for all integers $r \geq s$.

Proof. Theorems 1.1 and 2.3.

III. Stable range one for ring homomorphisms. A result of Evans [3, Theorem 2] states that any module whose endomorphism ring has 1 in its stable range may be cancelled from direct sums. (A ring $E$ has 1 in its stable range provided that whenever $f g+h=1$ in $E$, there exists $k \in E$ such that $f+h k$ is right invertible in $E$. It can be shown that this 
condition is left-right symmetric.) In fact, if $A$ is such a module, then given any module $M$ having internal direct sum decompositions $M=$ $A_{1} \oplus B_{1}=A_{2} \oplus B_{2}$ with $A_{1} \cong A_{2} \cong A$, there exists a submodule $C$ of $M$ such that $M=C \oplus B_{1}=C \oplus B_{2}$, and so the cancellation occurs because $B_{1} \cong M / C \cong B_{2}$. This so-called substitution property (the substitution of $C$ for the two copies of $A$ in such direct sum decompositions) is actually equivalent to the endomorphism ring of $A$ having 1 in its stable range [11, Theorem 2.1].

We obtain our cancellation results from these methods by simply changing module categories in mid-stream. For instance, to prove that a module $A$ has the property that whenever $B_{1}, B_{2}$ are modules satisfying $A \oplus B_{1} \cong A \oplus B_{2}$, then $n B_{1} \cong n B_{2}$ (for a pre-assigned positive integer $n$ ), it suffices to show that the diagonal map from the endomorphism ring of $A$ to the $n \times n$ matrix ring over the endomorphism ring of $A$ satisfies a suitable version of stable range 1 . (For a detailed development of these ideas in the case when $n$ is allowed to vary, see $[4,5]$.)

If a ring $E$ has 1 in its stable range, then it follows that all right or left invertible elements in $E$ are actually invertible (e.g., [11, Theorem 2.1]). Consequently, the condition that $E$ have 1 in its stable range may be restated in the form: $f g+h=1$ in $E$ implies that there exists $k \in E$ such that $f+h k$ is invertible. It is this latter version of the condition that we need to apply to ring homomorphisms.

Definition. Let $\varphi: E \rightarrow F$ be a ring homomorphism. (We are assuming that rings have units, and that ring homomorphisms are unital.) Let us say that 1 is in the stable range of $\varphi$ provided that for any $f, g, h \in E$ satisfying $f g+h=1$, there exists $k \in F$ such that $\varphi(f)+\varphi(h) k$ is invertible in $F$. (Strictly speaking, this condition should be called something like " 1 is in the strong stable range of $\varphi$ ", to distinguish it from the possibly weaker condition in which it is only required that $\varphi(f)+\varphi(h) k$ be right invertible in $F$.) To prove that this condition is left-right symmetric, we use the following result of Vasershtein, adapted from the proof of [10, Theorem 2]. We would like to thank R. K. Dennis for bringing this argument to our attention.

LEMMA 3.1. [Vasershtein] Let $a, b, c$ be elements of a ring $F$, such that $a b+c=1$. If there exists $x \in F$ such that $a+c x$ is invertible, then there exists $y \in F$ such that $b+y c$ is invertible.

Proof. Set $u=a+c x$, and set

$$
v=b+(1-b x) u^{-1} c \text { and } w=a+x(1-b a) .
$$


The lemma is established by showing that $v$ is invertible with inverse $w$.

First, observe that

$$
\begin{gathered}
v a=b a+(1-b x) u^{-1} c a \\
v x=b x+(1-b x) u^{-1}(u-a)=1-(1-b x) u^{-1} a \\
v x(1-b a)=(1-b a)-(1-b x) u^{-1}(1-a b) a \\
=1-b a-(1-b x) u^{-1} c a
\end{gathered}
$$

Adding equations (1) and (3) yields $v w=1$. Next, observe that

$$
\begin{gathered}
w b=a b+x b(1-a b)=a b+x b c, \\
\begin{aligned}
w(1-b x) & =a+x(1-b a)-a b x-x b c x \\
& =a+(1-a b) x-x b(a+c x) \\
& =a+c x-x b u=(1-x b) u,
\end{aligned} \\
w(1-b x) u^{-1} c=(1-x b) c .
\end{gathered}
$$

Adding equations (4) and (6) yields $w v=a b+c=1$.

Proposition 3.2. Let $\varphi: E \rightarrow F$ be a ring homomorphism. Then $\varphi$ has 1 in its stable range if and only if for any $f, g, h \in E$ satisfying $g f+h=1$, there exists $k \in F$ such that $\varphi(f)+k \varphi(h)$ is invertible in $F$.

Proof. First assume that $\varphi$ has 1 in its stable range. Given $f, g, h \in E$ with $g f+h=1$, there exists $k^{\prime} \in F$ such that $\varphi(g)+\varphi(h) k^{\prime}$ is invertible in $F$. Since $\varphi(g) \varphi(f)+\varphi(h)=1$, Lemma 3.1 shows that there exists $k \in F$ such that $\varphi(f)+k \varphi(h)$ is invertible in $F$. The converse is proved symmetrically.

In a similar manner, Lemma 3.1 may be used to show that the "power-substitution" condition studied in $[4,5]$ is left-right symmetric.

We use $\Re$ od- $R$ to denote the category of all right modules over a ring $R$.

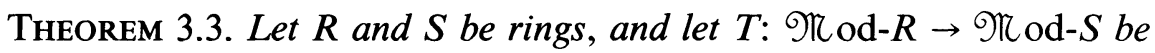
an additive functor. Let $A$ be a right $R$-module, and let

$$
\varphi: \operatorname{End}_{R}(A) \rightarrow \operatorname{End}_{S}(T(A))
$$


be the induced ring homomorphism, $e \mapsto T(e)$. Then $\varphi$ has 1 in its stable range if and only if the following condition holds:

$\left(^{*}\right)$ Given any direct sum decomposition $M=A_{1} \oplus B_{1}=A_{2} \oplus B_{2}$ of right $R$-modules with $A_{1} \cong A_{2} \cong A$, there exists an $S$-submodule $C$ of $T(M)$ such that

$$
T(M)=C \oplus T\left(B_{1}\right)=C \oplus T\left(B_{2}\right) .
$$

[In the statement of $\left({ }^{*}\right)$, we have identified $T\left(B_{i}\right)$ with the image of $T$ of the inclusion map $B_{i} \rightarrow M$. This is legitimate because $T$ preserves split monomorphisms.]

Proof. First assume that 1 is in the stable range of $\varphi$, and consider a right $R$-module direct sum decomposition

$$
M=A_{1} \oplus B_{1}=A_{2} \oplus B_{2}
$$

such that $A_{1} \cong A_{2} \cong A$. For $i=1,2$, let $r_{l}: A \rightarrow M$ be the composition of an isomorphism $A \rightarrow A_{i}$ with the inclusion map $A_{\imath} \rightarrow M$, and let $s_{i}$ : $B_{l} \rightarrow M$ be the inclusion map. There exist projection maps $p_{i}: M \rightarrow A$ and $q_{l}: M \rightarrow B_{t}$ such that

$$
p_{i} r_{i}=1_{A} ; \quad q_{i} s_{l}=1_{B_{i}} ; \quad p_{i} s_{i}=0 ; \quad q_{i} r_{i}=0
$$

for each $i$, and also $r_{\imath} p_{\imath}+s_{\imath} q_{i}=1_{M}$ for each $i$. (See Diagram I.)

$$
\begin{array}{cc}
A & A \\
r_{1} \downarrow \uparrow p_{1} & r_{2} \downarrow \uparrow p_{2} \\
A_{1} \oplus B_{1}=M=A_{2} \oplus B_{2} \\
s_{1} \uparrow \downarrow q_{1} & s_{2} \uparrow \downarrow q_{2} \\
B_{1} & B_{2} \\
\text { DIAGRAM I }
\end{array}
$$

Note that since

$$
T\left(p_{\imath}\right) T\left(s_{l}\right)=0 \quad \text { and } \quad T\left(r_{i}\right) T\left(p_{\imath}\right)+T\left(s_{i}\right) T\left(q_{i}\right)=1_{T(M)},
$$

we must have $\operatorname{ker}\left(T\left(p_{i}\right)\right)=\operatorname{im}\left(T\left(s_{l}\right)\right)=T\left(B_{i}\right)$.

Observe that

$$
1_{A}=p_{2} r_{2}=p_{2}\left(r_{1} p_{1}+s_{1} q_{1}\right) r_{2}=\left(p_{2} r_{1}\right)\left(p_{1} r_{2}\right)+\left(p_{2} s_{1} q_{1} r_{2}\right),
$$

and that the maps $p_{2} r_{1}, p_{1} r_{2}, p_{2} s_{1} q_{1} r_{2}$ all lie in $\operatorname{End}_{R}(A)$. As $\varphi$ has 1 in its stable range, there exists a map $k$ in $\operatorname{End}_{S}(T(A))$ such that the map

$$
\varphi\left(p_{2} r_{1}\right)+\varphi\left(p_{2} s_{1} q_{1} r_{2}\right) k=T\left(p_{2} r_{1}\right)+T\left(p_{2} s_{1} q_{1} r_{2}\right) k
$$


is an automorphism of $T(A)$. Set

$$
f=T\left(r_{1}\right)+T\left(s_{1} q_{1} r_{2}\right) k: T(A) \rightarrow T(M),
$$

so that $T\left(p_{2}\right) f$ is an automorphism of $T(A)$. Thus if $C=f(T(A))$, then

$$
T(M)=C \oplus \operatorname{ker}\left(T\left(p_{2}\right)\right)=C \oplus T\left(B_{2}\right) .
$$

In addition, $T\left(p_{1}\right) f=T\left(p_{1} r_{1}\right)=1_{T(A)}$, and hence

$$
T(M)=C \oplus \operatorname{ker}\left(T\left(p_{1}\right)\right)=C \oplus T\left(B_{1}\right),
$$

so that $\left(^{*}\right)$ is proved.

Conversely, assume that $\left({ }^{*}\right)$ holds, and consider maps $f, g, h \in$ End $_{R}(A)$ satisfying $f g+h=1$. Set $A_{1}=B_{1}=A$ and $M=A_{1} \oplus B_{1}$. Let

$$
p_{1}: M \rightarrow A_{1} ; \quad q_{1}: M \rightarrow B_{1} ; \quad r_{1}: A_{1} \rightarrow M ; \quad s_{1}: B_{1} \rightarrow M
$$

be the projection and injection maps for this direct sum. Define maps

$$
p_{2}=f p_{1}+h q_{1}: M \rightarrow A, \quad r_{2}=r_{1} g+s_{1}: A \rightarrow M,
$$

and note that $p_{2} r_{2}=f g+h=1_{A}$. Thus if $A_{2}=r_{2}(A)$ and $B_{2}=\operatorname{ker}\left(p_{2}\right)$, then $M=A_{2} \oplus B_{2}$ with $A_{2} \cong A$.

By (*), there exists an $S$-submodule $C$ of $T(M)$ such that

$$
T(M)=C \oplus T\left(B_{1}\right)=C \oplus T\left(B_{2}\right) .
$$

As $C$ and $T\left(A_{1}\right)$ are each complements for $T\left(B_{1}\right)$ in $T(M)$, we must have

$$
C \cong T(M) / T\left(B_{1}\right) \cong T\left(A_{1}\right)=T(A) .
$$

Consequently, there exists a monomorphism $t: T(A) \rightarrow T(M)$ such that $t(T(A))=C$. Since each of the maps $T\left(p_{i}\right): T(M) \rightarrow T(A)$ is a (split) epimorphism, and since

$$
T(M)=C \oplus T\left(B_{i}\right)=t(T(A)) \oplus \operatorname{ker}\left(T\left(p_{i}\right)\right),
$$

we see that $T\left(p_{i}\right) t$ is an automorphism of $T(A)$. Now

$$
T\left(p_{2}\right) t=T(f) T\left(p_{1}\right) t+T(h) T\left(q_{1}\right) t
$$

and hence

$$
\left[T\left(p_{2}\right) t\right]\left[T\left(p_{1}\right) t\right]^{-1}=T(f)+T(h)\left[T\left(q_{1}\right) t\right]\left[T\left(p_{1}\right) t\right]^{-1} .
$$

Therefore the map $k=\left[T\left(q_{1}\right) t\right]\left[T\left(p_{1}\right) t\right]^{-1}$ is an element of $\operatorname{End}_{S}(T(A))$ such that $\varphi(f)+\varphi(h) k$ is invertible in $\operatorname{End}_{S}(T(A))$, which proves that $\varphi$ has 1 in its stable range. 


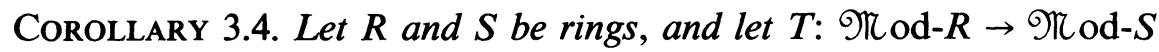
be an additive functor. Let $A$ be a right $R$-module and assume that the induced ring homomorphism

$$
\operatorname{End}_{R}(A) \rightarrow \operatorname{End}_{S}(T(A))
$$

has 1 in its stable range. If $B_{1}$ and $B_{2}$ are any right $R$-modules such that $A \oplus B_{1} \cong A \oplus B_{2}$, then $T\left(B_{1}\right) \cong T\left(B_{2}\right)$.

Proof. We may assume that there exists a right $R$-module $M$ with direct sum decompositions

$$
M=A_{1} \oplus B_{1}=A_{2} \oplus B_{2}
$$

such that $A_{1} \cong A_{2} \cong A$. By Theorem 3.3 , there is an $S$-submodule $C$ of $T(M)$ such that

$$
T(M)=C \oplus T\left(B_{1}\right)=C \oplus T\left(B_{2}\right) .
$$

Therefore $T\left(B_{1}\right) \cong T(M) / C \cong T\left(B_{2}\right)$.

IV. Vector bundle cancellation results. We apply Corollary 3.4 to certain modules of sections of vector bundles to obtain our cancellation results for vector bundles. We remind the reader of the notation $n \xi$ for the direct sum of $n$ copies of a vector bundle $\xi$.

THEOREM 4.1. Let $X$ be a paracompact topological space, let $\lambda, \xi_{1}, \xi_{2}$ be real vector bundles over $X$, and assume that $\lambda$ is a line bundle of finite type. If $\lambda \oplus \xi_{1} \cong \lambda \oplus \xi_{2}$, then:

(a) $\xi_{1}^{\mathbf{C}} \cong \xi_{2}^{\mathbf{C}}$ (as complex vector bundles).

(b) $2 \xi_{1} \cong 2 \xi_{2}$ (as real vector bundles).

Proof. As (b) follows immediately from (a), we need only prove (a).

Set $R=C(X, \mathbf{R})$ and $S=C(X, \mathbf{C})$, and let $T:$ Rod- $R \rightarrow$ श od- $S$ be the complexification functor $(-) \otimes_{\mathbf{R}}$ C. Set $A=\Gamma(\lambda)$ and $B_{i}=\Gamma\left(\xi_{i}\right)$ (for $i=1,2)$. Then $A \oplus B_{1} \cong A \oplus B_{2}$, and in view of Theorem 1.1 and Proposition 1.2, it suffices to show that $T\left(B_{1}\right) \cong T\left(B_{2}\right)$.

Let $\varphi: \operatorname{End}_{R}(A) \rightarrow \operatorname{End}_{S}(T(A))$ be the ring homomorphism induced by $T$. We claim that $\varphi$ has 1 in its stable range. Since $\lambda$ is a real line bundle of finite type, Proposition 1.3 shows that the natural ring homomorphism of $R$ into $\operatorname{End}_{R}(A)$ is an isomorphism. Similarly, as $\lambda^{\mathbf{C}}$ is a complex line bundle of finite type, the natural ring homomorphism of $S$ 
into $\operatorname{End}_{S}(T(A))$ is an isomorphism. Consequently, we obtain a commutative diagram as follows:

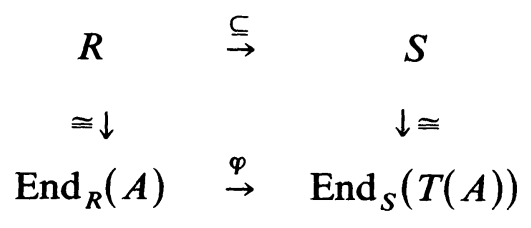

\section{Diagram II}

Hence, to prove that $\varphi$ has 1 in its stable range, it suffices to prove the same for the inclusion map $R \rightarrow S$.

Thus, consider any $f, g, h$ in $R$ satisfying $f g+h=1$. Then $f$ and $h$ are real-valued functions which do not simultaneously vanish at any point of $X$. Consequently, the complex-valued function $f+i$ never vanishes, and so $f+i h$ is an invertible element of $S$. This shows that the inclusion map $R \rightarrow S$ has 1 in its stable range and, hence, $\varphi$ does also.

As $A \oplus B_{1} \cong A \oplus B_{2}$, we can now conclude from Corollary 3.4 that $T\left(B_{1}\right) \cong T\left(B_{2}\right)$.

The second conclusion of Theorem 4.1 was noted (for the case that $X$ is compact) in [5, Theorem 14], as a consequence of the observation that the diagonal map from $C(X, \mathbf{R})$ to the $2 \times 2$ matrix ring over $C(X, \mathbf{R})$ has 1 in its stable range.

In proving a complex analog of Theorem 4.1, there is an obstacle, due to the fact that the complex field is not contained in the center of the quaternions. Namely, given an arbitrary complex line bundle $\lambda$ over $X$ of finite type, the endomorphism ring of $\Gamma(\lambda)$ can be identified with $C(X, \mathbf{C})$, but there is no natural way of identifying the endomorphism ring of $\Gamma\left(\lambda^{\mathbf{H}}\right)$ with $C(X, \mathbf{H})$; in fact, it is not even clear whether the endomorphism ring of $\Gamma\left(\lambda^{\mathbf{H}}\right)$ is necessarily isomorphic to $C(X, \mathbf{H})$. This difficulty can be circumvented in case $\lambda$ is a trivial vector bundle of rank 1 , or, more generally, if $\lambda$ is the complexification of a real line bundle.

THEOREM 4.2. Let $X$ be a paracompact topological space, let $\lambda, \xi_{1}, \xi_{2}$ be complex vector bundles over $X$, and assume that $\lambda$ is the complexification of a real line bundle of finite type. (In particular, $\lambda$ could be trivial of rank 1.) If $\lambda \oplus \xi_{1} \cong \lambda \oplus \xi_{2}$, then:

(a) $\xi_{1}^{\mathbf{H}} \cong \xi_{2}^{\mathbf{H}}$ (as quaternionic vector bundles).

(b) $\xi_{1} \oplus \bar{\xi}_{1} \cong \xi_{2} \oplus \bar{\xi}_{2}$ (as complex vector bundles). 
Proof. Set $R=C(X, \mathbf{C})$ and $S=C(X, \mathbf{H})$, and let $A=\Gamma(\lambda)$. As in Theorem 4.1, the desired conclusions follow from Corollary 3.4 provided that we show that the induced ring homomorphism from $\operatorname{End}_{R}(A)$ to End $_{S}\left(A \otimes_{\mathbf{C}} \mathbf{H}\right)$ has 1 in its stable range.

By assumption, there exists a real line bundle $\lambda_{0}$ over $X$ of finite type such that $\lambda_{0}^{\mathrm{C}}=\lambda$. Set $Q=C(X, \mathbf{R})$ and $B=\Gamma\left(\lambda_{0}\right)$. By Proposition 1.2, we may identify $A$ with $B \otimes_{\mathrm{R}} \mathbf{C}$, and then $A \otimes_{\mathrm{C}} \mathrm{H}$ becomes identified with $B \otimes_{\mathrm{R}} \mathbf{H}$. Remember that $B \otimes_{\mathrm{R}} \mathbf{C}$ and $B \otimes_{\mathrm{R}} \mathbf{H}$ are to be identified with $B \otimes_{Q} R$ and $B \otimes_{Q} S$, and that $B \otimes_{Q} S$ is a right $S$-module. Thus the problem now is to show that the induced ring homomorphism $\varphi$ from End $_{R}\left(B \otimes_{Q} R\right)$ to $\operatorname{End}_{S}\left(B \otimes_{Q} S\right)$ has 1 in its stable range.

According to Proposition 1.3, the natural ring homomorphism from $R$ to $\operatorname{End}_{R}\left(B \otimes_{Q} R\right)$ is an isomorphism. Under this isomorphism, an element $x \in R$ corresponds to the endomorphism sending any $b \otimes r$ to $b \otimes x r$. Since the ring $Q$ is the center of the ring $S$, we may define a ring homomorphism

$$
\psi: S \rightarrow \operatorname{End}_{S}\left(B \otimes_{Q} S\right)
$$

so that $\psi(y)(b \otimes s)=b \otimes y s$ for all $b \in B$ and all $y, s \in S$. We now have a commutative diagram as follows:

$$
\begin{array}{ccc}
R & \stackrel{\subseteq}{\rightarrow} & S \\
\cong \downarrow & & \downarrow \psi \\
\operatorname{End}_{R}\left(B \otimes_{Q} R\right) & \stackrel{\varphi}{\rightarrow} & \operatorname{End}_{S}\left(B \otimes_{Q} S\right)
\end{array}
$$

\section{DiAGRAM III}

To show that $\varphi$ has 1 in its stable range, it suffices to show that the inclusion map $R \rightarrow S$ does (since the composition of this map with $\psi$ will then have 1 in its stable range).

However, this is as easy as before. Given $f, g, h$ in $R$ satisfying $f g+h=1$, then $f$ and $h$ are complex-valued functions which do not simultaneously vanish at any point of $X$. Consequently, the quaternionvalued function $f+j h$ never vanishes, and so $f+j h$ is invertible in $S$. Therefore the inclusion map $R \rightarrow S$ does indeed have 1 in its stable range.

COROLlaRY 4.3. Let $X$ be a paracompact topological space, let $\lambda, \xi_{1}, \xi_{2}$ be real vector bundles over $X$, and assume that $\lambda$ is a direct sum of two line bundles of finite type. If $\lambda \oplus \xi_{1} \cong \lambda \oplus \xi_{2}$, then:

(a) $\xi_{1}^{\mathbf{H}} \cong \xi_{2}^{\mathbf{H}}$ (as quaternionic vector bundles). 
(b) $\xi_{1}^{\mathbf{C}} \oplus \overline{\xi_{1}^{\mathbf{C}}} \cong \xi_{2}^{\mathbf{C}} \oplus \overline{\xi_{2}^{\mathbf{C}}}$ (as complex vector bundles).

(c) $4 \xi_{1} \cong 4 \xi_{2}$ (as real vector bundles).

Proof. By assumption, $\lambda=\lambda_{1} \oplus \lambda_{2}$ for some line bundles $\lambda_{1}, \lambda_{2}$ of finite type. Then

$$
\lambda_{1} \oplus\left(\lambda_{2} \oplus \xi_{1}\right) \cong \lambda_{1} \oplus\left(\lambda_{2} \oplus \xi_{2}\right) .
$$

Applying Theorem 4.1, we obtain $\lambda_{2}^{\mathbf{C}} \oplus \xi_{1}^{\mathbf{C}} \cong \lambda_{2}^{\mathrm{C}} \oplus \xi_{2}^{\mathrm{C}}$. Then Theorem 4.2 applies, yielding $\left(\xi_{1}^{\mathbf{C}}\right)^{\mathbf{H}} \cong\left(\xi_{2}^{\mathbf{C}}\right)^{\mathbf{H}}$, or $\xi_{1}^{\mathbf{H}} \cong \xi_{2}^{\mathbf{H}}$. The remaining conclusions follow immediately.

The last conclusion of Corollary 4.3 may be derived directly from Corollary 3.4, using the fact that the inclusion map from the $2 \times 2$ matrix ring over $C(X, \mathbf{R})$ into the $2 \times 2$ matrix ring over $C(X, \mathbf{H})$ has 1 in its stable range. (The proof of this fact requires a certain amount of matrix manipulation.)

Theorems 4.1 and 4.2, and Corollary 4.3, also hold for smooth vector bundles over a smooth manifold $X$, since the inclusion maps from $C^{\infty}(X, \mathbf{R})$ to $C^{\infty}(X, \mathbf{C})$ and from $C^{\infty}(X, \mathbf{C})$ to $C^{\infty}(X, \mathbf{H})$ each have 1 in their stable range.

It might seem plausible to try proving one further result of the type we have derived by using the Cayley numbers. However, as our method applies to endomorphism rings, which are necessarily associative, there is no way for the Cayley numbers to fit into our method.

We would like to raise the question of cancellation results of this type for higher-rank vector bundles, at least in the real case. Here is one possible suggestion.

Problem. Let $X$ be a paracompact topological space, let $\lambda, \xi_{1}, \xi_{2}$ be real vector bundles over $X$, and assume that $\lambda$ is trivial of rank $n$. If $\lambda \oplus \xi_{1} \cong \lambda \oplus \xi_{2}$, does it follow that $2 n \xi_{1} \cong 2 n \xi_{2}$ ? [The answer is positive in case $n=1$ or 2 , by Theorem 4.1 and Corollary 4.3 , and the answer is also positive in case $\xi_{2}$ is trivial, by Lam's result (Corollary 2.2).]

\section{REFERENCES}

[1] H. Bass, Algebraic K-Theory, New York (1968) Benjamin.

[2] N. Bourbaki, Commutative Algebra, Paris/Reading (1972) Hermann/Addison-Wesley.

[3] E. G. Evans, Jr., Krull-Schmidt and cancellation over local rings, Pacific J. Math., 46 (1973), 115-121.

[4] K. R. Goodearl, Power-cancellation of groups and modules, Pacific J. Math., 64 (1976), 387-411. 
[5] Power-cancellation of modules, in Ring Theory II, Proceedings of the Second Oklahoma Conference (B. R. McDonald and R. A. Morris, Eds.), pp. 131-147, New York (1977) Dekker.

[6] W. Greub, S. Halperin, and R. Vanstone, Connections, Curvature, and Cohomology, Volume I, Pure and Applied Math. Series, Vol. 47-I, New York (1972), Academic Press.

[7] D. Husemoller, Fibre Bundles, New York (1966), McGraw-Hill.

[8] T. Y. Lam, Series summation of stably free modules, Quart. J. Math. (Oxford), (2) 27 (1976), 37-46.

[9] R. G. Swan, Vector bundles and projective modules, Trans. Amer. Math. Soc., 105 (1962), 264-277.

[10] L. N. Vasershtein, Stable rank of rings and dimensionality of topological spaces, Functional Anal. Appl., 5 (1971), 102-110.

[11] R. B. Warfield, Jr., Cancellation of modules and groups and stable range of endomorphism rings, Pacific J. Math., 91 (1980), 457-485.

Received December 2, 1982.

UNIVERSITY OF UTAH

SAlt LAKe City, UT 84112 


\section{PACIFIC JOURNAL OF MATHEMATICS \\ EDITORS}

Donald BaBBITT (Managing Editor)

University of California

Los Angeles, CA 90024

Hugo RossI

University of Utah

Salt Lake City, UT 84112

C. C. Moore and Arthur Ogus

University of California

Berkeley, CA 94720
J. DugundiI

Department of Mathematics

University of Southern California

Los Angeles, CA 90089-1113

R. FINN and H. SAMELSON

Stanford University

Stanford, CA 94305

\section{ASSOCIATE EDITORS}
R. ARENS
E. F. BECKENBACH
B. H. NeUMANN
F. WOLF
K. YoshidA (1906-1982)

\section{SUPPORTING INSTITUTIONS}

\author{
UNIVERSITY OF ARIZONA \\ UNIVERSITY OF BRITISH COLUMBIA \\ CALIFORNIA INSTITUTE OF TECHNOLOGY \\ UNIVERSITY OF CALIFORNIA \\ MONTANA STATE UNIVERSITY \\ UNIVERSITY OF NEVADA, RENO \\ NEW MEXICO STATE UNIVERSITY \\ OREGON STATE UNIVERSITY
}

\author{
UNIVERSITY OF OREGON \\ UNIVERSITY OF SOUTHERN CALIFORNIA \\ STANFORD UNIVERSITY \\ UNIVERSITY OF HAWAII \\ UNIVERSITY OF TOKYO \\ UNIVERSITY OF UTAH \\ WASHINGTON STATE UNIVERSITY \\ UNIVERSITY OF WASHINGTON
}

The Supporting Institutions listed above contribute to the cost of publication of this Journal, but they are not owners or publishers and have no responsibility for its content or policies.

Mathematical papers intended for publication in the Pacific Journal of Mathematics should be in typed form or offset-reproduced (not dittoed), double spaced with large margins. Please do not use built up fractions in the text of the manuscript. However, you may use them in the displayed equations. Underline Greek letters in red, German in green, and script in blue. The first paragraph must be capable of being used separately as a synopsis of the entire paper. In particular it should contain no bibliographic references. Please propose a heading for the odd numbered pages of less than 35 characters. Manuscripts, in triplicate, may be sent to any one of the editors. Please classify according to the scheme of Math. Reviews, Index to Vol. 39. Supply name and address of author to whom proofs should be sent. All other communications should be addressed to the managing editor, or Elaine Barth, University of California, Los Angeles, California 90024.

There are page-charges associated with articles appearing in the Pacific Journal of Mathematics. These charges are expected to be paid by the author's University, Government Agency or Company. If the author or authors do not have access to such Institutional support these charges are waived. Single authors will receive 50 free reprints; joint authors will receive a total of 100 free reprints. Additional copies may be obtained at cost in multiples of 50 .

The Pacific Journal of Mathematics is issued monthly as of January 1966. Regular subscription rate: $\$ 132.00$ a year (6 Vol., 12 issues). Special rate: $\$ 66.00$ a year to individual members of supporting institutions.

Subscriptions, orders for numbers issued in the last three calendar years, and changes of address should be sent to Pacific Journal of Mathematics, P.O. Box 969, Carmel Valley, CA 93924, U.S.A. Old back numbers obtainable from Kraus Periodicals Co., Route 100, Millwood, NY 10546.

The Pacific Journal of Mathematics ISSN 0030-8730 is published monthly by the Pacific Journal of Mathematics at P.O. Box 969, Carmel Valley, CA 93924. Application to mail at Second-class postage rates is pending at Carmel Valley, California, and additional mailing offices. Postmaster: Send address changes to Pacific Journal of Mathematics, P. O. Box 969, Carmel Valley, CA 93924.

PUBLISHED BY PACIFIC JOURNAL OF MATHEMATICS, A NON-PROFIT CORPORATION

Copyright $\odot 1984$ by Pacific Journal of Mathematics 


\section{Pacific Journal of Mathematics}

\section{Vol. 113, No. $2 \quad$ April, 1984}

Alan Adolphson, On the Dwork trace formula ...................257

Amos Altshuler and Leon Steinberg, Enumeration of the quasisimplicial

3 -spheres and 4-polytopes with eight vertices .................. 269

Kenneth R. Goodearl, Cancellation of low-rank vector bundles .......... 289

Gary Fred Gruenhage, Ernest A. Michael and Yoshio Tanaka, Spaces

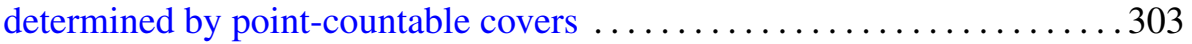

Charles Lemuel Hagopian, Atriodic homogeneous continua .......... 333

David Harbater, Ordinary and supersingular covers in characteristic $p$. . . 349

Domingo Antonio Herrero, Continuity of spectral functions and the lakes

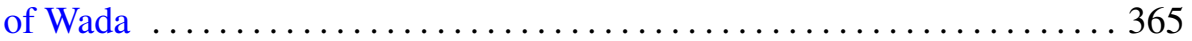

Donald William Kahn, Differentiable approximations to homotopy resolutions and framed cobordism ....................... 373

K. McGovern, On the lifting theory of finite groups of Lie type $\ldots \ldots \ldots . \ldots 383$

C. David (Carl) Minda, The modulus of a doubly connected region and the geodesic curvature-area method ............................. 395

Takuo Miwa, Complexes are spaces with a $\sigma$-almost locally finite base $\ldots . .407$

Ho Kuen Ng, Finitely presented dimension of commutative rings and modules

Roger David Nussbaum, A folk theorem in the spectral theory of

$C_{0}$-semigroups

J. S. Okon, Prime divisors, analytic spread and filtrations

Harold Raymond Parks, Regularity of solutions to elliptic isoperimetric problems

R. Sitaramachandra Rao and M. V. Subba Rao, Transformation formulae for multiple series

Daniel Ruberman, Imbedding punctured lens spaces and connected sums

Uri Srebro, Deficiencies of immersions 